C.F.H.T. FOCAL REDUCER : IMAGE

AND MULTIAPERTURE SPECTROSCOPY

B. Fort ${ }^{1}$, G. Lelièvre ${ }^{2}$, J.P. Picat ${ }^{1}, Y, R^{3}{ }^{3}, L$. Vigroux ${ }^{3}$

Sunmary :

In this poster paper we shortly present recent developpment and preliminary results obtained with the focal reducer of the Canada French Hawai 3.6 meter telescope with new trends for deep imagery and multiaperture spectroscopy.

\title{
Focal Reducer
}

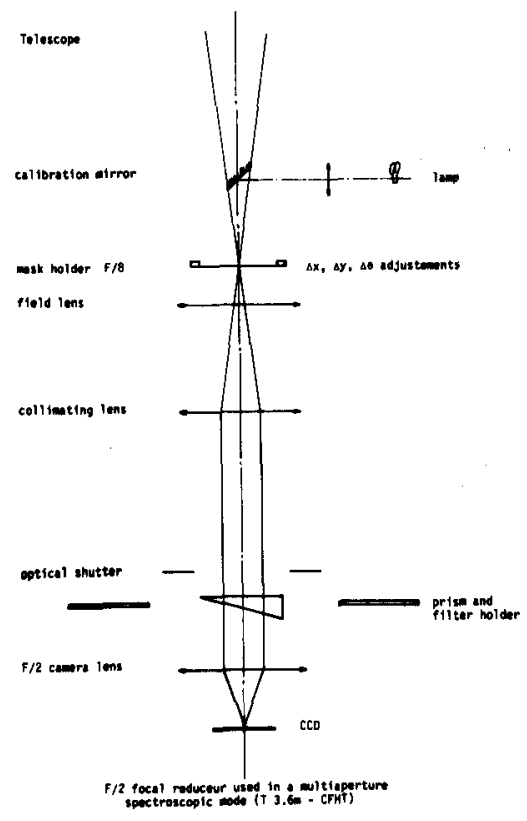

Designed (fig. 1) by Marseille observatory to study the cinematic of galaxies with Perot Fabry (ref. Courtes, 1960. Boulesteix et al 83), this focal reducer is a perfect match for the pixel size of CCD Cameras for photometry of very faint isolated objects. $F / 2$ aperture gives a linear scale of 30 microns for the 0.8 arc second regular seeing. This figures minimize the surface lecture noise of CCD and provide a very fast instrument for deep imagery and multiaperture spectroscopy.

Fig. 1 (left) : Optical design of the focal reducer

Detection of Faint Point source

For example it can be used for the detection and $B, V, R, I$ photometry of point object like: optical conterpart of radiogalaxies, XuV or $\gamma$ sources or for distant cluster of galaxies (fig. 2). The photometry is generally obtained from the external part of a stars cluster. The accordance with photoelectric measurement give a r.m.s. accuracy within 0.03 magnitude.

1 - Observatoire de Toulouse, 1 avenue Edouard Belin, 31400 Toulouse

2 - Canada-France-Hawai Telescope

3 - C.E.N. Saclay, Gif/Yvette B.P. $n^{*} 2$ - 91 France

Proceedings of the IAU Colloquium No. 79: "Very Large Telescopes, their Instrumentation and Programs", Garching, April 9-12, 1984. 


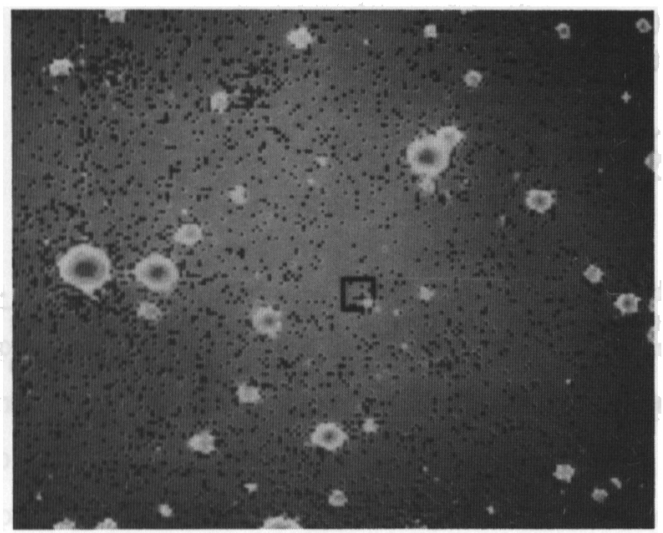

Fig. 2 (left) : An identification for "Geminga" (2CG 195 + 04) : the optical counterpart of IE 0630 + 78. R Filter, $10 \mathrm{mn}$ exposure time. 3.6 meter CFH Telescope. Image obtained by L. Vigroux, April 83 (A. Caraveo et al 84 ).

Detection and photometry of very faint extended objects
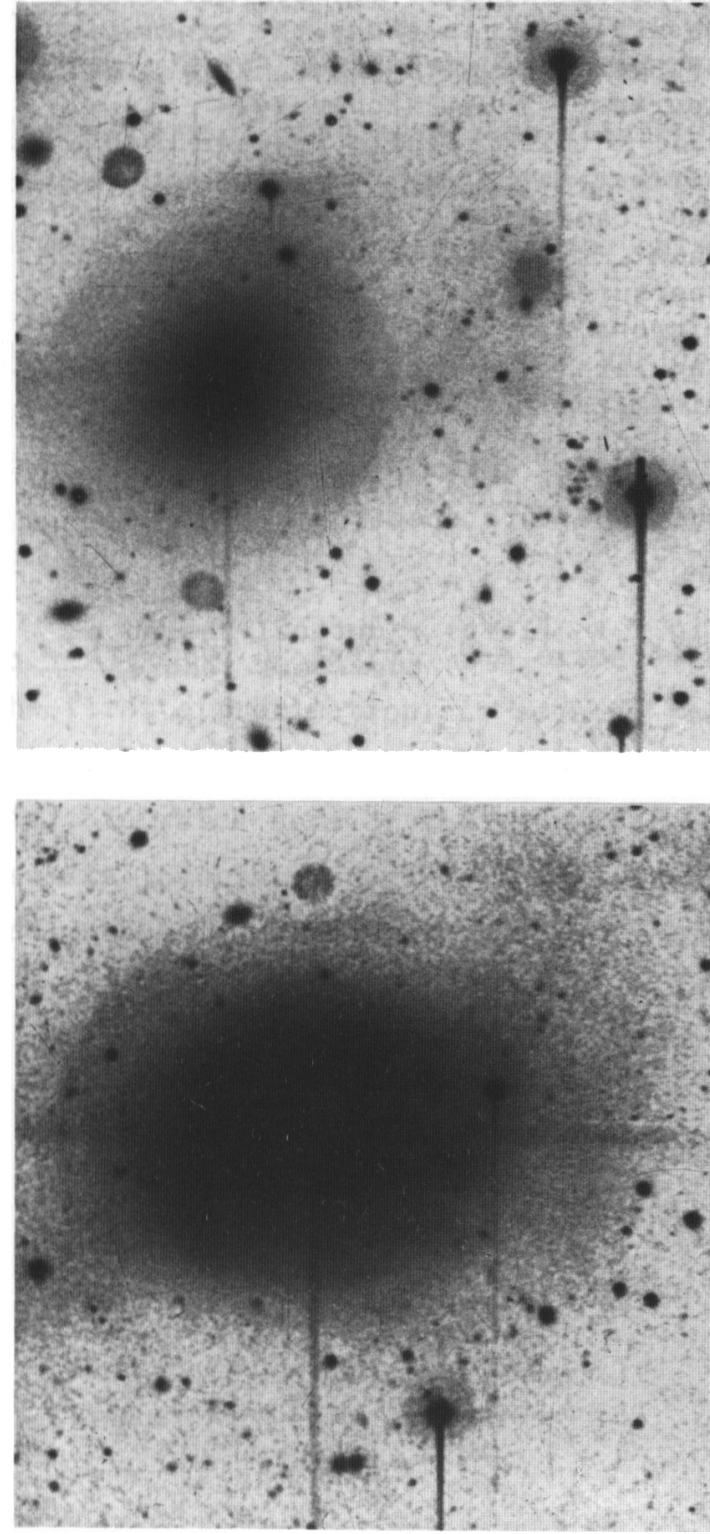

The focal reducer is fast and very effective for the photometry of faint surface objects. The galactic shells of NGC 2865 are given as an exemple on fig. 3. The image was obtained with a $30 \mathrm{mn}$ exposure time with a green filter $1210 \AA$ bandpass avoiding the bright sky line OI 5576 A) (D. Carter, B. Fort and L. Vigroux, CFHT April B3). A radial cut throught the shell (white arrow) indicates the magnitude per square arc sec.

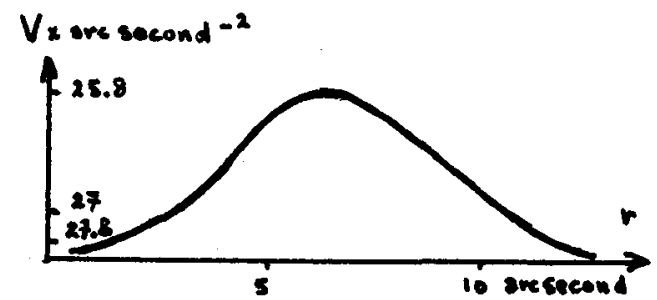

These shells are generally bluer than the central part of the $E$ galaxy but can be seen in $B, V, R$ and I. According to numerical simulation these structures may represent a phase wrapping process of a disc galaxy around the main elliptical galaxy louinn thesis 83). Image of NGC 5018, fig. 4, was obtained with a $10 \mathrm{mn}$ exposure and a $R$ filter.

Note on these pictures the ghost images of several bright stars $(m \approx 18)$ which saturate some $C C D$ columns (D. Carter, B. Fort, L. Vigroux CFHT April 83l. 


\section{Multiaperture spectroscopy}

The hunt for large redshift $Q S O$ and the study of distant cluster of galaxies required low resolution spectroscopy ( $\Delta \lambda$ about $5 \AA$ to $40 \AA$ ). Even with 4 meter class telescope and fast CCD the exposure time could be of several hours for a single spectra. It is then necessary to developp some multislit spectroscopy techniques (Dresler and Gunn 83 ). The focal reducer is known to be very well adapted for such a trend, and we have developped recently such a new procedure for the CFHT focal reducer described below :

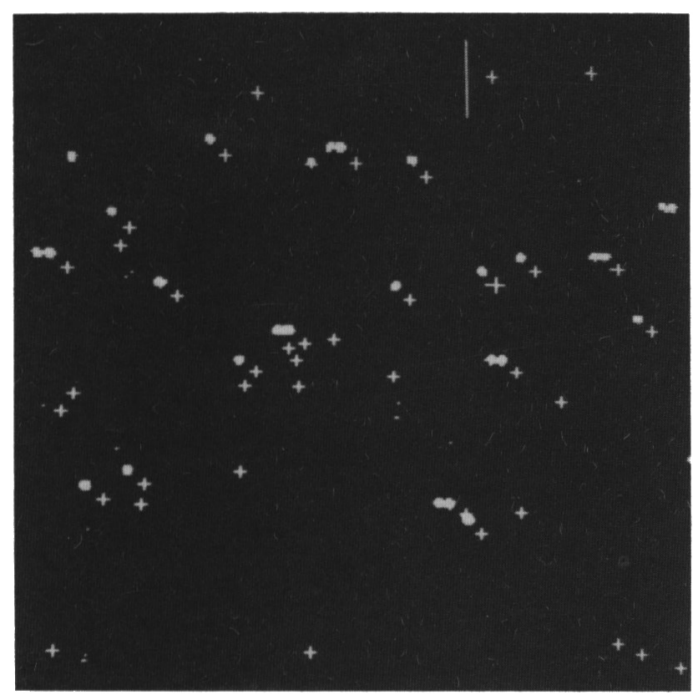

Fig. 5 : Simultaneous video display of the binary field image (crosses) and front-side illuminated apertures which are used for alignment of the mask central partl on the $256 \times 256$ pixel quick look video display)
1) A CCD image of the field is obtained with a short exposure (V or $R$ filter, $=5 \mathrm{mn}$ ). Accurate numerical coding position of an offset star is recorded on a guide probe.

2) The on-line computer displays a negative mask of the field on a plotter. Photograph of the mask is made on a Kodalithe orthofilm, type 3. The software adapts to the magnification, corrects residual optical distortion and applies various selection criteria : no superimposition of spectra; selection of sky reference apertures ; magnitude selection : $20.5<v<23 ;$ ghost image cleaning etc...

3) Mask is positioned with a simulteneous video display of field objects (binary images of crosses) and mask apeatures (front-side illuminated by the calibration Lamp, fig. 5). The true position is obtained in a few minutes when the two images are superimposed, using a microdisplacement $\Delta x, \Delta y, \Delta \theta$ of the mask

4) A multiaperture spectroscopic $C C D$ image is then recorded with a Carpenter prism. (Grism).

The first observational test was performed in March 84 18. Fort, $G$. Lelievre, $Y$. Rio, J.P. Picatl. The preliminary results show the possibility to record more than 50 spectra between 4000 and 8500 \& with spectral resolution between 40 to $8 \mathrm{~A} / \mathrm{pixel}$ and a usable aperture between 1.5 to 2.5 arcsecond. The magnitude attainable is $\left(m_{v}>19.5\right.$ up to 22. for exposure time of about 1 hourl.

Important improvements are in progress at Toulouse observatory; a mechanical device controlled by the computer will punch a metallic mask in actual time after the field CCD exposure. A paper describing. the technics in more details will be published very soon. 


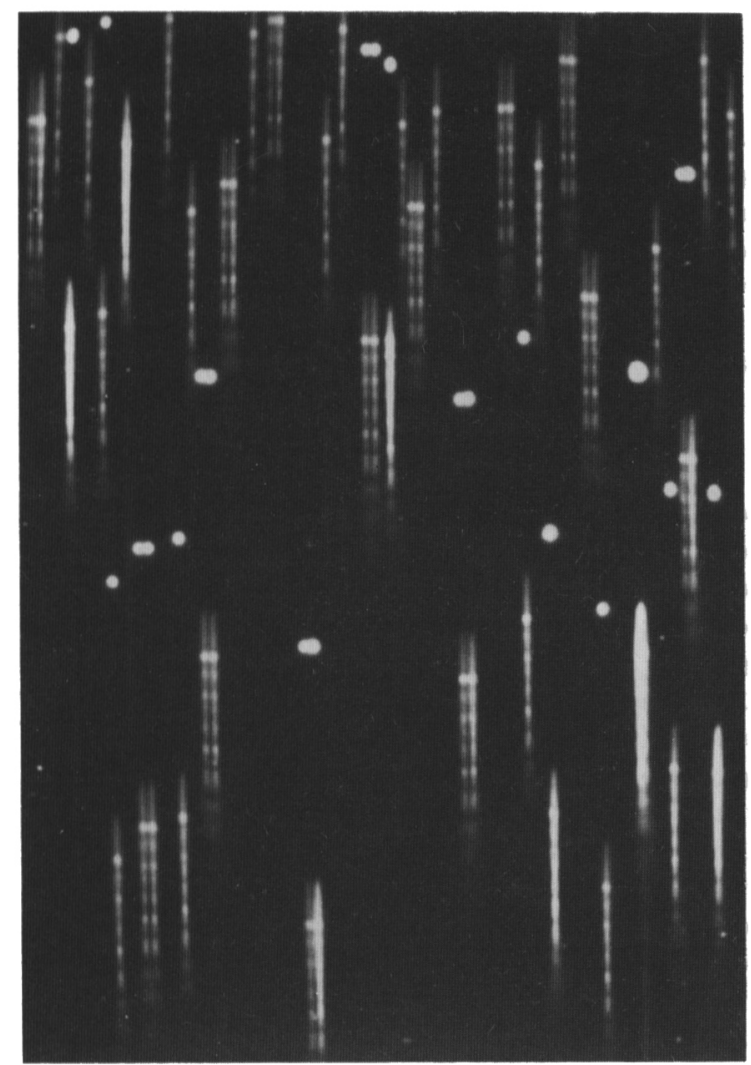

a

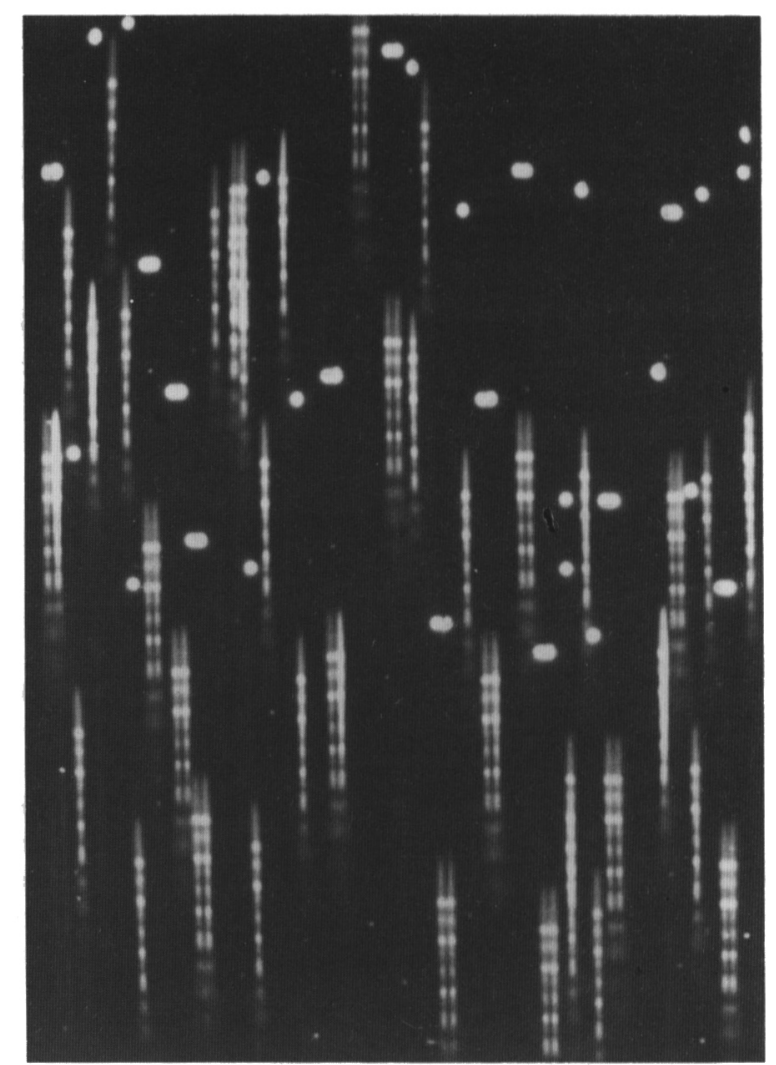

b

Fig. 6 : Multiaperture spectroscopy in the fields of $Q S O$ OH 471. 1 hour exposure time. Some sky reference spectra are automatically recorded in the vicinity of some faint objects. The aperture was 2.5 arc-second and dispersion $40 \AA \times$ pixel $^{-1}$ between $4500 \AA$ and $8500 \AA$. 16 a ; ler mask 39 objects + 14 sky, $6 b$ : $2 e$ mask ; 45 objects with 36 new objects plus 16 skyl.

\section{Bibliography}

J. Boulesteix, Y. Georgelin, M. Marcellin, G. Monnet 1983 Proc SPIE 445, Instrumentation in astronomy $V, A$ Boksenberg and $D$ Crawford editor.

P.A. Caraveo, G.F. Bignami, L. Vigroux and J.A. Paul, 1984 AP Letters 276 , p. 9

D. Carter, B. Fort, L. Vigroux 1983 private communication paper in preparation

G. Courtes, 1960, Ann. Astrophysics, 23, p. 115

A. Oressler and J. Gunn, 1983, Ap J 270 p. 7

\section{Aknowledgment}

We thank very much Or. M.H. Ulrich which give us the opportunity to present this work at the IAU colloquium $n^{\circ} 79$ just few weeks after the observationnal run. We are also very grateful to the technical staff of CFHT corporation, R. Jouan from CEN Saclay and $Y$. Georgelin from Marseilles observatory who help us on telescope. 